\title{
An Energy-efficient Data Transmission Scheme in Underwater Wireless Sensor Networks
}

\author{
Yousef Emami \\ Computer Engineering and IT Department \\ Shiraz University of Technology \\ Shiraz, Iran \\ yousef.emami@ieee.org
}

\author{
Reza Javidan \\ Computer Engineering and IT Department \\ Shiraz University Technology \\ Shiraz, Iran \\ javidan@sutech.ac.ir
}

\begin{abstract}
Energy is a precious resource in underwater wireless sensor networks (UWSNs). In these networks, the number of data transmissions between sensor nodes dominates energy consumption. Complex signal processing techniques also increase energy consumption. In this paper an energy-efficient data transmission scheme based on bloom filters is proposed. Extensive simulation is carried out to demonstrate the effectiveness of the proposed method. Simulation results indicate that the proposed scheme outperforms the primary technique in terms of energy efficiency, lifetime, load and loss rate. The results of this research suggest that exploiting bloom filters is a viable solution for reducing the number of transmissions in UWSNs.
\end{abstract}

Keywords-Energy Efficiency; Data Transmission; Bloom Filter; Underwater Wireless Sensor Networks

\section{INTRODUCTION}

Today, underwater remote sensing systems such as SONARs are used extensively for detecting and tracking of different targets using sound signals. High-resolution acoustic images obtained from these systems play an important role in underwater exploration [1]. However, these systems are costly and applying them calls for several considerations. Even though other methods, such as satellite marine observation for change detection, are also applicable [2], their applications are limited. Underwater Wireless Sensor Networks (UWSNs) have recently attracted significant attention due to the potential benefits for marine monitoring. They are cost effective and easy to use. It should be noted that there is a huge difference between UWSNs and terrestrial wireless sensor network (TWSNs). From the communication point of view, TWSNs utilize radio signal while UWSNs utilize underwater acoustic channels to transmit data Usually the network model of TWSNs is two-dimensional while that of UWSNs is threedimensional. The nodes in TWSNs can be replaced at any time but in UWSNs this process is cumbersome due to the type of environment and costs [3].

Hence, energy efficiency in UWSNs is of vital importance. UWSNs exploit complex signal processing techniques and support sparse deployment. Hence, energy consumption is higher than terrestrial sensors. The unique challenges of UWSNs are limited bandwidth, long propagation delay, high bit error rates, and limited battery power. Nevertheless,
UWSNs enjoy myriad of applications in different area, including environmental monitoring, undersea explorations, disaster prevention, navigation, distributed tactical surveillance and mine reconnaissance [4].

Many researches regarding saving energy in UWSNs have been carried out [5-9]. Some of them focused on clustering sensor nodes and optimizing routing protocols. Less works reported on data transmission problem. The developed energyefficient transmission schemes for TWSNs cannot be applied in underwater environment because of the challenges of acoustic communications mentioned above. As a result, novel transmission schemes have to be implemented for UWSNs.

In this paper, an energy-efficient data transmission scheme based on bloom filters in UWSNs is proposed. Data transmission is the main source of energy consumption in UWSNs. Data transmission in UWSNs is not cost effective. Hence, to improve energy efficiency and increase network lifetime, the cardinal rule is to diminish the number of transmission. The content of this paper follows this cardinal rule. The results of this research suggest that exploiting bloom filters is a viable solution for reducing the number of transmission in UWSNs. Simulations on prototype data were carried out to demonstrate the effectiveness of the proposed method. Simulation results showed that the proposed scheme outperforms other well-known method in term of energy efficiency, lifetime, load and loss rate.

\section{RELATED WORKS}

A novel FEC approach for multiple-path communication in UWSNs was proposed in [6]. To reduce the consumed energy of transmission, a Markov model is utilized. In addition, jettison retransmission, multiple-path communication, and hamming coding are integrated. A novel energy-efficient data transmission scheme for UWSNs called EGRC (Energyefficiency Grid Routing based on 3D Cubes) was proposed in [7], taking complex properties of underwater medium into consideration. In this method, the $3 \mathrm{D}$ network is divided into multiple parts and each part itself shapes a cluster. In essence, a new algorithm for cluster-head selection and a novel mechanism for seeking the next-hop node is proposed. A balance transmission mechanism, in which data transmission is divided in two phases was proposed in [9]. In the routing set-up 
phase, an efficient routing algorithm based on the optimum transmission distance is present to optimize the energy consumption of the UWASN. After that, in data transmission phase a balance transmission algorithm is introduced. In this method one-hop or multihop data transmission of the node to the sink node can be determined based on the energy level of adjacent nodes. Energy efficient transmission in UWSNs using optimization problem which aims to minimize energy consumption, was investigated in [10]. The results showed that reliability and communication delay are key factors for transmission. A fair data transmission strategy in UWSNs by comprising queue management, transmission control and the concept of time distance ratio, was proposed in [11]. Transmission control assigns a channel in each node queue based on the maximum time distance ratio. The algorithm considers the fairness of network performance and data transmission based on network congestion.

Bloom filters are widely used in the realm of network security. Wireless networks utilize bloom filters for authentication, anonymity and privacy-preserving firewalling, tracebacking, misbehavior detection, replay attack detection and node replication detection. Wired networks used bloom filters for string matching, IP tracebacking, spam filtering and email protection, DoS and DDoS attacks detection and anomaly detection [12]. Denh et al. [13] proposed a coordinated packet traceback protocol (CAPTRA) for wireless sensor networks. In CAPTRA each sensor dedicates a subtle memory for the bloom filter. When a packet passes through the network, each forwarding sensor records the packet in its bloom filter. Later the bloom filter will be used to rebuild the attack graph. Toe et al. [14] proposed a bloom filter based data dissemination protocol for wireless sensor networks. Utilizing bloom filters identifies the version difference among data items and guarantee network-wide consistency.

Compared with above-mentioned related works, the contribution of our paper is twofold: a novel approach based on bloom filter is proposed for improving energy efficiency and lifetime in UWSNs and extensive simulation experiments are conducted to evaluate the performance of the proposed method. The results indicate that this method can reduce network load and loss rate.

\section{THE PROPOSED METHOD}

\section{A. Bloom Filter}

Bloom filter is a simple and compact probabilistic data structure for representing a set of $\mathrm{n}$ elements and supporting membership queries. Bloom filter utilizes an array of $\mathrm{m}$ bits (initially all bits set to zero). In order to add an element to the bloom filter, the element is $\mathrm{k}$ times hashed, the results of hashing operation produces the array's indexes, then the corresponding bits of these indexes are set to one. In the rest of this paper such an operation is referred as bloom. To support membership query for a specific element like $\mathrm{y}$, it $\mathrm{k}$ times is hashed, if one of the corresponding bits of the obtained array's indexes is zero $y$ is not member of the set. If all of the corresponding bits of the obtained array's indexes are one, y is either in the set or is a false positive. Bloom filter may result in false positive, i.e. an element considered to be in the set though it is not. The probability of a false positive is determined by:

$$
\left(1-\left(1-\frac{1}{\mathrm{~m}}\right)^{\mathrm{kn}}\right)^{\mathrm{k}} \approx\left(1-\mathrm{e}^{\frac{\mathrm{kn}}{\mathrm{m}}}\right)^{\mathrm{k}}
$$

Where $\mathrm{m}$ is array's length, $\mathrm{n}$ is the number of element to be hashed and the value of $\mathrm{k}$ determines the number of hashing. In practice, it is better to deem $\mathrm{k}$ a small value since the amount of computation is reduced. For a graphical example of the process, the reader may refer to [14]. A counting bloom filter is a special version of bloom filters in which each entry in the bloom filter is a small counter instead of a single bit. Upon inserting an item the relevant counters are incremented and in case of deletion the corresponding counters are decremented [15].

\section{B. The Proposed Method}

The proposed method exploits 32 bit bloom filters to deliver maximum energy efficiency in underwater wireless sensor networks. The case study in this research is water quality monitoring. Each front-end sensor node senses data for the target features (ammonia, phosphorous, nitrogen and oxygen) in the determined time intervals and bloom the sensed data for each feature in a separate bloom, according the rules of counting bloom filters. Then the shaped bloom filters are sent towards the middle nodes. The middle nodes consolidate the received bloom filters from sensors and integrate them with its own sensed and bloomed data and the result are sent toward the sink using DBR routing protocol [16]. Figure 1 illustrates the above procedure using a numerical example. For the sake of simplicity just one target feature is depicted. In the end, the sink extracts data from the received bloom filters so that fuzzy logic may be employed to determine the final result. In this method, many packets are converted to one packet using bloom filter; hence the amount of traffic is reduced and energy efficiency improvement is achieved.

Based on the extracted data in the sink, a fuzzy logic based method is used to determine the quality of the water. Such a quality depends on the dissolved oxygen $\mathrm{O}_{2}$, total nitrogen $\mathrm{N}$, ammonia NH3-N and total phosphorus $\mathrm{P}$.

One of the best features of using fuzzy logic is that it does not require complex mathematical equation and system modeling [17]. Therefore, lower cost and better performance is achieved. Figure 2 shows the proposed fuzzy logic inference system. Using fuzzy logic, the input crisp values should be fuzzified. The fuzzified values are then processed by the inference engine. The knowledge base of the inference engine consists of a series of IF-THEN rules. Here inferencing on the rules are carried on based on the Mamdani method. Finally the output fuzzy values should be defuzzified to generate a crisp output.

As shown in Figure 2, the proposed fuzzy approach consists of four input parameters $\mathrm{O}_{2}, \mathrm{~N}, \mathrm{NH} 3-\mathrm{N}$ and $\mathrm{P}$, and one output, with universal of discourse $[0 \ldots 19],[0 \ldots 100],[0 \ldots 45],[0 \ldots 30]$ and $[0 \ldots 100]$, respectively. The proposed method uses five membership function for each input and output parameters as shown in Figure 3. Table I shows some of the IF-THEN rules 
used in the fuzzy approach. As an example, IF $\mathrm{O}_{2}$ is high, $\mathrm{P}$ is low, $\mathrm{N}$ is low and NH3-N is high, THEN output is clear. In the end the defuzzification finds a crisp output value. Defuzzification is done using centre-of-maxia method [18] given by:

$$
\text { Outpout }=\frac{x_{1} \mu_{1}+x_{2} \mu_{2}+\ldots+x_{n} \mu_{n}}{\mu_{1}+\mu_{2}+\ldots+\mu_{n}}
$$

where $x_{n}$ is the numerical value and $\mu_{n}$ is the degree of membership.

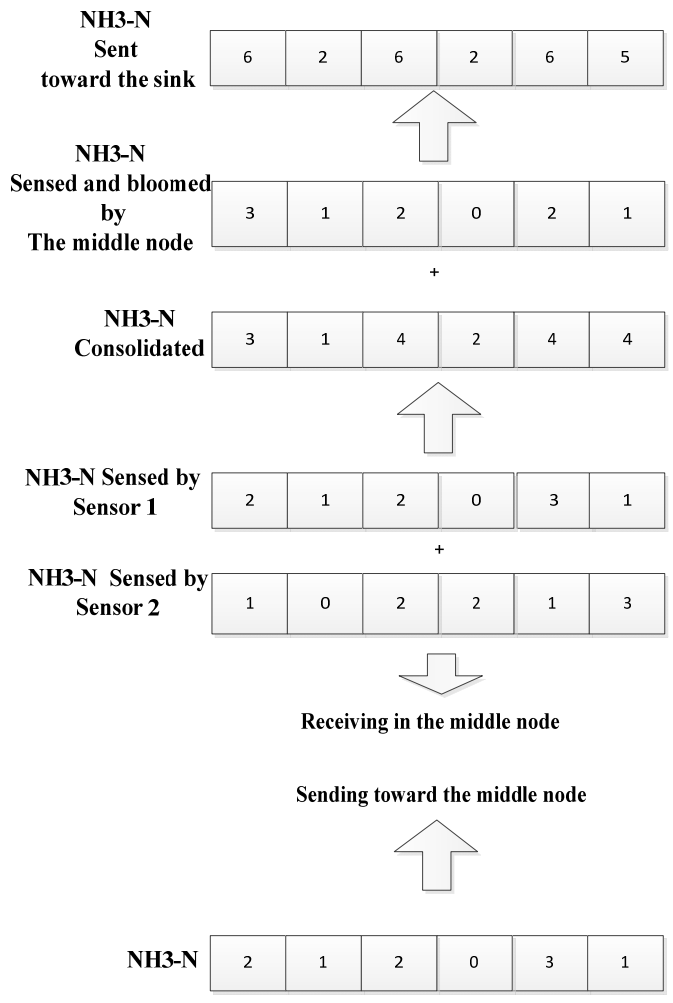

Fig. 1. A Numerical example for bloom filter in the proposed approach

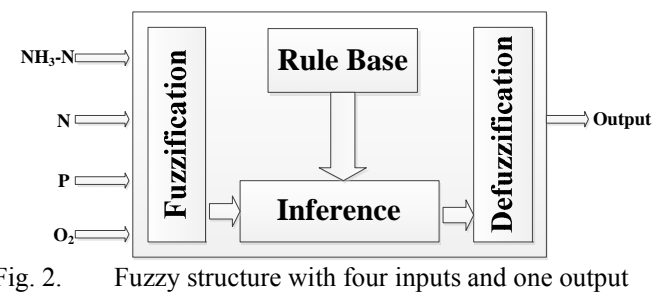

IV. PERFORMANCE EVALUATION

To show the effectiveness of the proposed method, the method is compared with the well known primary method. In primary method, no blooming is occurred, each front-end node sense data in the determined times and send them toward the middle nodes, then the middle nodes receive and buffer these data, in special times the buffered data is extracted and sent for sink using DBR. It is notable that the performance of the proposed method is evaluated from two points of view. Initially, the effect of sensing intervals on the proposed method is checked and finally the effect of gathering intervals is investigated. In data gathering intervals the middle nodes bloom the received packets and send them toward sink. Finally in sensing interval, the front-end nodes and also the middle nodes sense the chemical features of water.
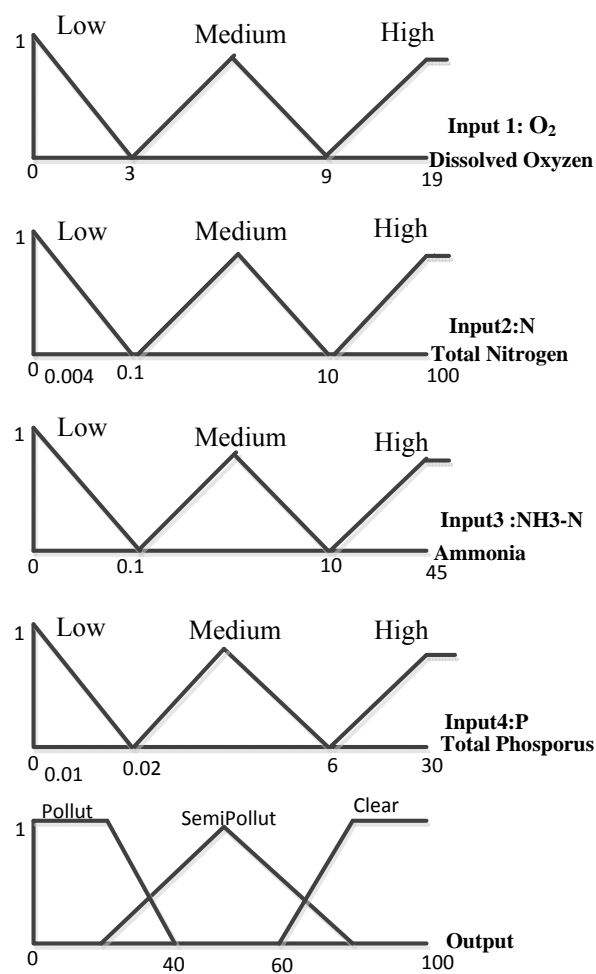

Fig. 3. Membership graph for the inputs and the output

TABLE I. IF-THEN RULES

\begin{tabular}{|c|c|c|c|c|c|}
\hline \multirow[t]{2}{*}{ No } & \multicolumn{4}{|c|}{ Antecedent } & \multirow{2}{*}{$\begin{array}{c}\text { Consequent } \\
\text { Result }\end{array}$} \\
\hline & $\mathbf{O}_{2}$ & $\mathbf{P}$ & $\mathbf{N}$ & NH3-N & \\
\hline 1 & $\mathrm{~L}$ & $\mathrm{~L}$ & $\mathrm{~L}$ & $\mathrm{~L}$ & SEMI P \\
\hline 2 & $\mathrm{~L}$ & $\mathrm{~L}$ & $\mathrm{~L}$ & $\mathrm{H}$ & SEMI P \\
\hline 3 & $\mathrm{~L}$ & $\mathrm{~L}$ & $\mathrm{H}$ & $\mathrm{L}$ & SEMI P \\
\hline 4 & $\mathrm{~L}$ & $\mathrm{~L}$ & $\mathrm{H}$ & $\mathrm{H}$ & $\mathrm{P}$ \\
\hline 5 & $\mathrm{~L}$ & $\mathrm{H}$ & $\mathrm{L}$ & $\mathrm{L}$ & SEMI P \\
\hline 6 & $\mathrm{~L}$ & $\mathrm{H}$ & $\mathrm{L}$ & $\mathrm{H}$ & $\mathrm{P}$ \\
\hline 7 & $\mathrm{~L}$ & $\mathrm{H}$ & $\mathrm{H}$ & $\mathrm{L}$ & $\mathrm{P}$ \\
\hline 8 & $\mathrm{~L}$ & $\mathrm{H}$ & $\mathrm{H}$ & $\mathrm{H}$ & $\mathrm{P}$ \\
\hline 9 & $\mathrm{H}$ & $\mathrm{L}$ & $\mathrm{L}$ & $\mathrm{L}$ & $\mathrm{C}$ \\
\hline 10 & $\mathrm{H}$ & $\mathrm{L}$ & $\mathrm{L}$ & $\mathrm{H}$ & $\mathrm{C}$ \\
\hline 11 & $\mathrm{H}$ & $\mathrm{L}$ & $\mathrm{H}$ & $\mathrm{L}$ & $\mathrm{C}$ \\
\hline 12 & $\mathrm{H}$ & $\mathrm{L}$ & $\mathrm{H}$ & $\mathrm{H}$ & SEMI P \\
\hline 13 & $\mathrm{H}$ & $\mathrm{H}$ & $\mathrm{L}$ & Low & $\mathrm{C}$ \\
\hline 14 & $\mathrm{H}$ & $\mathrm{H}$ & $\mathrm{L}$ & $\mathrm{H}$ & SEMI P \\
\hline
\end{tabular}

Simulations are carried out in Aqua-Sim. Aqua-Sim is based on NS-2 and simulates the attenuation of underwater acoustic channels [19]. The utilized MAC and routing protocol are broadcast MAC and DBR respectively. The initial energy of sensors is 20J. Two hundred nodes are scattered randomly in 
a $1000 * 1000$ area with depth of $500 \mathrm{~m}$. There is only one sink. The energy consumption parameters are set according to the UWM2000 LinkQuest Underwater Acoustic Modem [20]. Detailed simulation parameters are listed in Table II.

TABLE II. SIMULATION PARAMETERS

\begin{tabular}{|c|c|}
\hline MAC Protocol & Broadcast MAC \\
\hline Routing Protocol & DBR \\
\hline Simulation Time & 2000 Second \\
\hline Bandwidth & $17.8 \mathrm{kbps}$ \\
\hline Contamination Indicator & 10 \\
\hline Number of repetitions for each scenario & 200 \\
\hline Number of Nodes & $1000 \mathrm{~m} * 1000 \mathrm{~m}$ \\
\hline Investigated Area & $500 \mathrm{~m}$ \\
\hline Depth(meters) & LinkQuest2000uwm \\
\hline Sensor type & 20 Joule \\
\hline Initial energy of node & 50 second \\
\hline Sensing Interval for pollution indicators & 20 \\
\hline Data processing interval & 25 to 45 second \\
\hline The Number of front-end Nodes & \\
\hline $\begin{array}{c}\text { The interval for data gathering ,saving and } \\
\text { sending in the middle nodes }\end{array}$ & \\
\hline
\end{tabular}

In data processing intervals, data are processed using fuzzy logic. All obtained results in the figures have been repeated 10 times and their average have been presented. The calculated confidence interval is 95 percent. The utilized performance metrics are energy efficiency, life time, network load and loss rate. In the following a short description of each metric is presented.

- Energy efficiency: energy efficiency is expressed as

$$
\frac{\text { Packet Delivery Ratio }}{\text { Energy Consumption }}
$$

where packet delivery ratio is the number of received packet in the sink.

- Life time: The time until at least 10 percent of the nodes are drained of their energy.

- Network load: refers to the amount of data (traffic) being carried by the network.

- Loss Rate: The amount of lost information by the network (transmitted data not delivered to destination due to collision). In other word loss rate is equal to 1-packet delivery ratio.

\section{A. Performance with Sensing Interval Changing}

In this part the impact of changing the sensing intervals on the performance of the proposed method is investigated. To obtain the following results, sensing and sending intervals vary from 3 to 15 seconds, gathering interval in the middle nodes is 20 seconds and fuzzy processing interval is 50 seconds. As it can be seen in Figure 4(a), the proposed method shows an improvement from energy efficiency point of view compared to the primary method. This is because the proposed method combines multiple packets into one packet using bloom filters in the middle nodes thus energy consumption is reduced and energy efficiency is improved. Figure 4(b) depicts that the proposed method has longer lifetime than the primary method, since the number of transmitted packets in the middle nodes is reduced. Therefore energy consumption by each sensor is diminished. Lower energy consumption in sensor results in increasing the overall lifetime. The amount of generated traffic for the proposed method is clearly less than the primary method; traffic load for the proposed method is as shown in Figure 4(c). Figure 4(d) indicates that the loss rate for the proposed method is less than its primary counterpart. Obviously decreasing the number of transferred packets reduces the number of collisions.

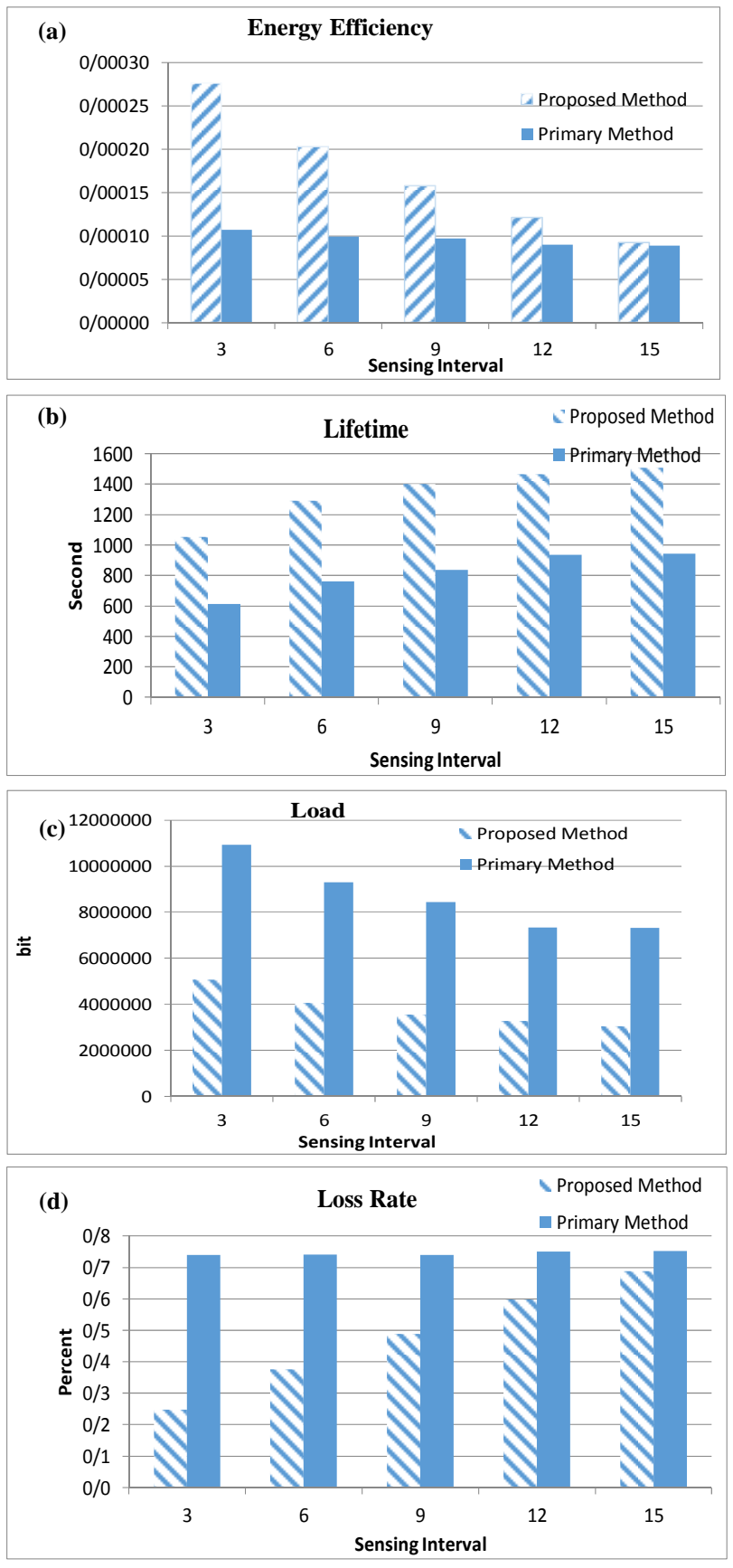

Fig. 4. Simulation results for sensing interval 

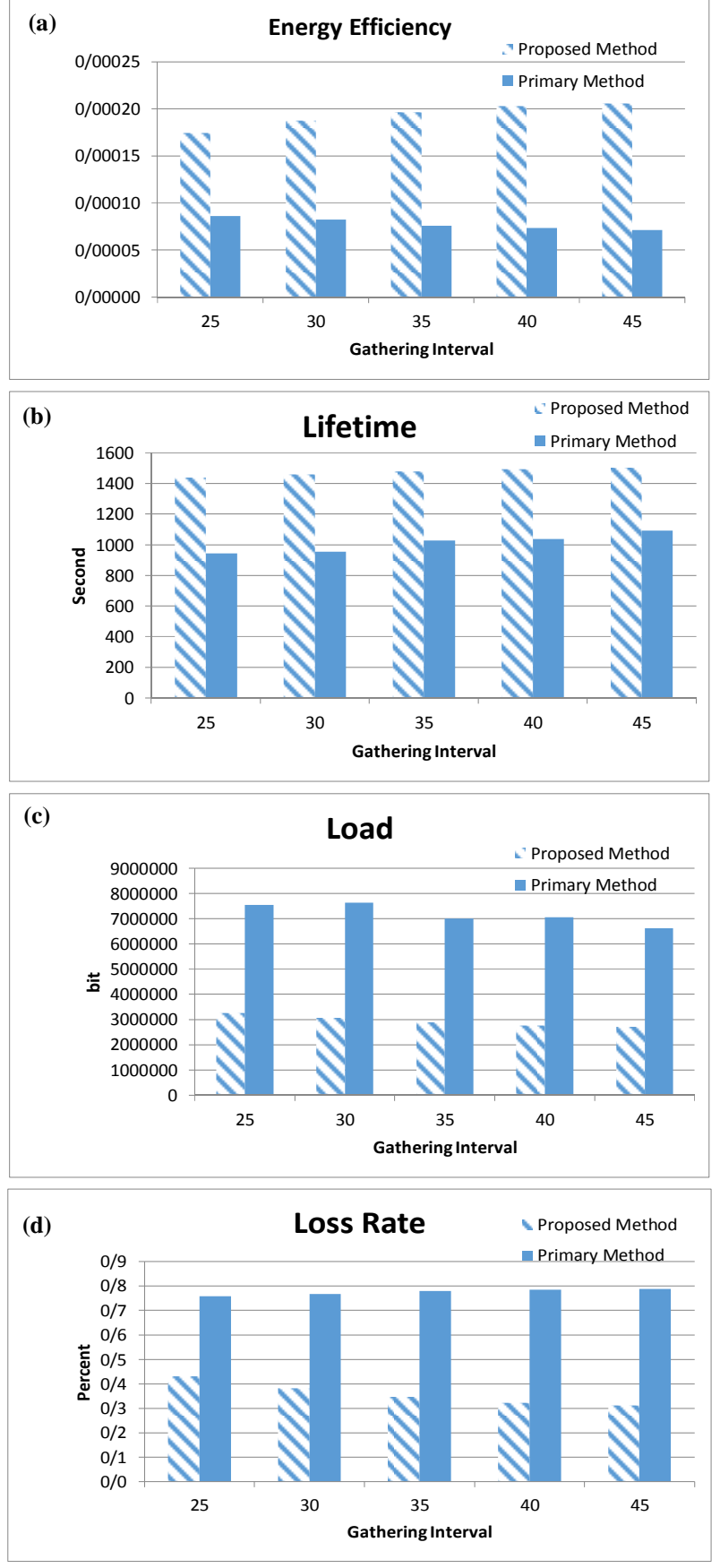

Fig. 5. Simulation results for gathering interval

\section{B. Performance with Gathering Interval Changing}

Figure 4 investigates the impact of sensing interval on the protocol performance. Figure 5 investigate the impact of buffering time in the middle nodes on the protocol performance. To obtain the results, sensing interval is set to 9 second, gathering interval in the middle nodes vary from 25 to 45 seconds and fuzzy processing interval is 50 seconds. As Figure 5(a) illustrates, the proposed method outperform the primary one from energy efficiency point of view. The reason behind this is summarizing packets in the middle nodes using bloom filters. As gathering intervals in the figure step towards the end, the middle nodes have more time for buffering the received packets and blooming them, hence energy efficiency is greatly improved. Reducing the number of transmissions results in increased lifetime. Figure 5(b) clearly demonstrates that the proposed method has better performance in this regard. As shown in Figure 5(c), it is clear that the volume of generated traffic in the proposed method is less than that of the primary method since the proposed method utilize bloom filter for data summarizing which reduces traffic load. Figure 5(d) illustrates the improved performance for the proposed method in term of loss rate. The reason is that sending less number of packets will result less collision

\section{CONCLUSION}

In this paper, a novel energy-efficient data transmission scheme using bloom filters for UWSNs is proposed.. Transmission and computation are the two main sources of energy consuming in UWSNs but the energy required for computation is subtler than that of transmission. To save the precious energy in UWSNs the best feasible option is to cut the number of transmissions with cost effective solutions. Bloom filters with compact data structure can be a good selection. The proposed method utilize bloom filters to efficiently cut the number of transmissions and to prolong the overall lifetime of the network. Extensive simulations on Aqua-Sim showed that the proposed approach can significantly outperform the primary method in terms of energy efficiency, lifetime, and load and loss rate

\section{ACKNOWLEDGMENT}

Yousef Emami would like to express his gratitude to Dr. Michele Nati, Senior Researcher at the University of Surrey, for persevering with him as mentor through out the time it took him to complete this research and write the dissertation.

\section{REFERENCES}

[1] R. Javidan, M. A. Masnadi-Shirazi, Z. Azimifar, "Contourlet-Based Acoustic Seabed Ground Discrimination System", Third IEEE International Conference on Information and Communication Technologies: From Theory to Applications, Damascus, Syria, April 711,2008

[2] T. J. Arsanjani, R. Javidan, M. J. Nazemosadat, J. J. Arsanjani, E Vaz, "Spatiotemporal monitoring of Bakhtegan Lake's areal fluctuations and an exploration of its future status by applying a cellular automata model", Computers \& Geosciences, No. 78, pp. 37-43, 2015.

[3] V. Kavoosi, M. J. Dehghani, R. Javidan, "Selective geometry for nearfield three-dimensional localisation using one-pair sensor", IET Radar, Sonar \& Navigation (accepted)

[4] R. Mohammadi, S. Y. Nabavi, R. Javidan, "MAC Protocols in Underwater Wireless Sensor Networks: Issues and simulations", Journal of Advances in Computer Research, Vol. 5, No. 2, pp. 97-109, 2014

[5] S. Rowshanrad, M. Keshtgary, R. Javidan, "MBC: A Multi-hop Balanced Clustering Routing Protocol for Wireless Sensor Networks", International Journal of Artificial Intelligence and Mechatronics, Vol. 2, No. 6, pp. 164-170, 2014.

[6] J. Xu, K. Li, G. Min, "Reliable and Energy-Efficient Multipath Communications in Underwater Sensor Networks", IEEE Transactions on Parallel and Distributed Systems, vol. 23, No. 7, pp. 1326-1335, 2012

[7] K. Wang, H.Gao, X.Xu, and D.Yue, "An Energy-efficient Reliable Data Transmission Scheme for Complex Environmental Monitoring in 
Underwater Acoustic Sensor Networks," IEEE Sensors Journal, , vol. PP, pp. 1-1, 2015.

[8] D. Pompili, T. Melodia, I. F. Akyildiz, "Distributed Routing Algorithms for Underwater Acoustic Sensor Networks", IEEE Transactions on Wireless Communications, Vol. 9, No. 9, pp. 2934-2944, 2010

[9] J. Cao, J. Dou, S. Dong, "Balance Transmission Mechanism in Underwater Acoustic Sensor Networks", International Journal of Distributed Sensor Networks, Vol. 2015, Article ID 429340, pp. 1-12, 2015

[10] H. Yang, B. Liu, F. Ren, H. Wen, C. Lin, “Optimization of Energy Efficient Transmission in Underwater Sensor Networks", IEEE Global Telecommunications Conference, pp. 1-6, Honolulu, Nov. 30-Dec. 4, 2009

[11] C. Yuan, X. Wang, S. Xiong, L. Ma, Q. Miu, “A Fair Data Transmission Strategy in Underwater Acoustic Sensor Networks", Journal of Networks, Vol. 9, No. 10, pp. 2606-2614, 2014

[12] S. Geravand, M. Ahmadi, "Bloom filter applications in network security: A state-of-the-art survey", Computer Networks, Vol. 57, No. 18, pp. 4047-4064, 2013

[13] D. Sy, L. Bao, “CAPTRA: coordinated packet traceback”, The Fifth International Conference on Information Processing in Sensor Networks, IPSN 2006, Nashville, USA, pp. 152-159, 2006
[14] T. Chen, D. Guo., Y. He, H. Chen, X. Liu, X. Luo, "A Bloom filters based dissemination protocol in wireless sensor networks", Ad Hoc Networks, Vol. 11, No. 4, pp. 1359-1371, 2013

[15] A. Broder, M. Mitzenmacher, "Network Applications of Bloom Filters: A Survey", Internet Mathematics, Vol. 1, No. 4, pp. 485-509, 2003

[16] R. Mohammadi, R. Javidan, A. Jalili, "Fuzzy depth based routing protocol for underwater acoustic wireless sensor networks", Journal of Telecommunication, Electronic and Computer Engineering", Vol. 7, No. 1,pp. 81-86, 2015

[17] A. S. Gad, "A fuzzy logic-based multisensor data fusion for Maritime Surveillance”, National Radio Science Conference, pp. 1-10, New Cairo, Egypt, March 17-19, 2009.

[18] T. A. Runkler, "Selection of appropriate defuzzification methods using application specific properties", IEEE Transactions on Fuzzy Systems, Vol. 5, No. 1, pp. 72-79, 1997

[19] X. Peng, "Aqua-Sim: An NS-2 based simulator for underwater sensor networks", OCEANS 2009, MTS/IEEE Biloxi - Marine Technology for Our Future: Global and Local Challenges, Biloxi, USA, pp. 1-7, 26-29 Oct. 2009

[20] LinkQuest Inc, LinkQuest Underwater Acoustic Modems UWM2000 Specifications, available at: http://www.link-quest.com/html/ 